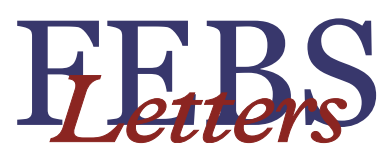

journal homepage: www.FEBSLetters.org

\title{
K153R polymorphism in myostatin gene increases the rate of promyostatin activation by furin
}

\author{
György Szláma, Mária Trexler, László Buday, László Patthy* \\ Institute of Enzymology, Research Centre for Natural Sciences, Hungarian Academy of Sciences, P.O. Box 286, Budapest H-1519, Hungary
}

\section{A R T I C L E I N F O}

\section{Article history:}

Received 21 August 2014

Revised 27 November 2014

Accepted 12 December 2014

Available online 25 December 2014

Edited by Berend Wieringa

\section{Keywords:}

Aging

Lifespan

Longevity

Muscle strength

Myostatin

Obesity

\begin{abstract}
A B S T R A C T
Recent studies demonstrated an association between the K153R polymorphism in the myostatin gene with extreme longevity, lower muscle strength and obesity but the molecular basis of these associations has not been clarified. Here, we show that the K153R mutation significantly increases the rate of proteolysis of promyostatin by furin, but has no effect on the activity of the latent complex or the cleavage of the latent complex by bone morphogenetic protein 1 (BMP-1). The increased rate of activation of K153R mutant promyostatin may explain why this polymorphism is associated with obesity, lower muscle strength and extension of lifespan.
\end{abstract}

(c) 2014 The Authors. Published by Elsevier B.V. on behalf of the Federation of European Biochemical Societies. This is an open access article under the CC BY-NC-ND license (http://creativecommons.org/ licenses/by-nc-nd/4.0/).

\section{Introduction}

Myostatin, similarly to other members of the TGF- $\beta$ family, is secreted as a precursor protein, promyostatin [1]. Two molecules of promyostatin are covalently linked via a single disulfide bond present in the C-terminal growth factor domain. Mature myostatin is liberated from promyostatin by proteolytic processing. In the first step of myostatin activation furin type proprotein convertases cleave both chains of the homodimeric precursor at the boundary of the prodomain and the growth factor domain. The two prodomains and the disulfide-bonded growth factor domains remain associated, forming a non-covalent complex [2], usually referred to as a latent complex. Mature growth factor is liberated from this latent complex through degradation of the prodomain by proteases of the BMP-1/Tolloid family of metalloproteinases [3].

Myostatin is a negative regulator of skeletal muscle growth: mice lacking myostatin are characterized by a dramatic increase in skeletal muscle mass [4]. Mutations in the myostatin gene that

Abbreviations: BMP, bone morphogenetic protein; BSA, bovine serum albumin; FBS, fetal bovine serum; MSTN, myostatin gene; PMSF, phenylmethylsulfonyl fluoride; TGF, transforming growth factor

* Corresponding author at: Budapest, Magyar tudósok körútja 2, H-1117, Hungary.

E-mail addresses: szlama.gyorgy@ttk.mta.hu (G. Szláma), trexler.maria@ttk.mta. hu (M. Trexler), buday.laszlo@ttk.mta.hu (L. Buday), patthy.laszlo@ttk.mta.hu (L. Patthy). interfere with the formation of mature myostatin were also shown to cause hypermuscularity in mice and cattle [5-8]. A mutation in the myostatin gene leading to mis-splicing and loss of myostatin protein was shown to cause muscle hypertrophy in a human child [9].

Myostatin is also involved in regulation of adipose tissue: in $\mathrm{Mstn}^{-/-}$mice adipose tissue size is greatly reduced [10-11]. The positive effects of myostatin inhibition on adipose tissue seem to be indirect results of the changes of muscle mass and muscle metabolism [12].

Human genomic studies have identified several missense substitutions in the coding region of the human myostatin gene affecting conserved amino acid residues: A55T, K153R, E164K, P198A, and I225T [13]. Two of these, A55T and K153R, were shown to be polymorphic in the general population [13].

Several recent studies suggest that the most common polymorphism K153R may have significant functional consequences. Saunders et al. [14] provided compelling evidence that mutation K153R has been subject to recent positive selection, suggesting that it is associated with functional differences.

Recent studies indicate that the K153R mutation may have three major types of phenotypic consequences: on muscle, on adipose tissue and on lifespan.

In a cohort of older women significant association was found with the maintenance of muscle strength and the K153R polymorphism: the R153 allele was associated with lower muscle strength 
[15]. In nonagerians heterozygosity for the K153R polymorphism had no negative influence on the muscle phenotypes of women, but the woman bearing the RR genotype had very low values of muscle mass and functional capacity [16]. In a study with non athletic young men, Santiago et al. [17] found that the K153R polymorphism affects the ability to produce 'peak' power during muscle contractions: men with the KR genotype had a worse performance in vertical jumps compared with those with the wildtype KK genotype [17].

The K153R variant of myostatin was found to predispose to obesity in Asian Indians: the RR genotype of the K153R polymorphism was found to be associated with a high risk of obesity [18].

The K153R polymorphism was also shown to be associated with longevity [19]: in a Spanish cohort of centenarians the study of the genotype distributions of the exonic myostatin variants showed that the frequency of the $\mathrm{R}$ allele of the K153R polymorphism was significantly higher in centenarians $(7.1 \%)$ than in controls (2.7\%). These results were independently confirmed in an Italian study, where a higher frequency of the R allele was found in centenarians (7.6\%) compared to controls (3.0\%), indicating that the variant allele of the myostatin K153R polymorphism could be among the genetic contributors associated with exceptional longevity. These authors suggested that the beneficial effect of the K153R mutation on longevity is based on the inhibitory role that myostatin plays in the mTOR signaling pathway.

In summary, the most common polymorphism of the human myostatin gene, the K153R polymorphism, shows significant association with lower muscle strength and obesity. Since this is just the opposite of what we observe in the case of mutations leading to loss of myostatin activity, it seemed plausible to assume that the K153R mutation might increase myostatin activity. To address this question, in the present work we have studied the molecular properties of recombinant myostatin precursor carrying the K153R mutation and compared its properties with those of the wild-type protein. Our studies have shown that the K153R substitution significantly increases the rate of furin cleavage but has no effect on the activity of latent myostatin or its cleavage by BMP1. Since latent myostatin is known to have significant activity [20], an increase in the amount of furin-activated myostatin and concomitant increase in myostatin activity may explain the observed association of K153R mutation with lower muscle strength and obesity.

We suggest that the association of the K153R mutation with extreme longevity is also explained by increased myostatin activity. It is noteworthy in this respect that the myostatin ortholog of Drosophila, myoglianin, has been recently shown to control longevity: its overexpression in muscle extends lifespan and delays systemic aging of flies by acting on muscle and adipocytes [21], raising the possibility that myostatin may also regulate lifespan in mammals [22].

\section{Materials and methods}

2.1. Reagents, enzymes, $P C R$ primers, proteins, bacterial strains, cell lines, and media

Mutagenesis primers were from Integrated DNA Technologies (Coralville, IA, USA). The mutagenesis reaction was performed with Quickchange mutagenesis kit (Agilent Technologies, Inc. Santa Clara, CA USA). DNA was purified with Nucleospin Extract DNA purification kit (Macherey-Nagel, Duren, Germany). Escherichia coli JM109 was used for DNA propagation during DNA manipulation steps, and E. coli BL21(DE3) strain was used for protein expression.

For the Luciferase reporter assay the SBE4 SMAD responsive firefly luciferase plasmid was from Addgene (Addgene plasmid 16495; www.addgene.org, Cambridge, MA, USA). Rhabdomyosarcoma
A204 and HEK 293 cells were purchased from German Collection of Microorganisms and Cell Cultures (DSMZ, Braunschweig, Germany); Fugene HD transfection reagent was from Promega (Promega Corporation, Madison, WI, USA). DMEM and McCoy's 5A culture medium and heat-inactivated fetal bovine serum were from Invitrogen (Carlsbad, CA, USA).

Recombinant human promyostatin was produced in E. coli as described previously [20]. Recombinant furin, recombinant BMP1, anti-Mouse Myostatin Propeptide sheep polyclonal antibody (AF1539) (R\&D Systems, Minneapolis, MN, USA), alkaline phosphatase conjugated anti-sheep antibody (Sigma-Aldrich, St Louis, MO, USA) and Furin Inhibitor I (Merck Millipore, Darmstadt, Germany) were commercial products. Mammalian expression vectors of the Stargate system, pENTRY-IBA51 and pCSG-IBA142, were purchased from IBA BioTAGnology (Göttingen, Germany).

\subsection{Production of the K153R mutant human promyostatin in Escherichia coli}

Mutagenesis was performed with QuikChange Site-Directed Mutagenesis, using the $5^{\prime}$ CAA TAC AAT AAA GTA GTA AGG GCC CAA CTA TGG ATA TAT $3^{\prime}$ sense and 5' ATA TAT CCA TAG TTG GGC CCT TAC TAC TTT ATT GTA TTG 3'antisense primers. The expression plasmid pPR-IBA2_Promyo containing the cDNA of human promyostatin [20] was the template in the mutagenesis reaction. The mutagenesis introduced an $A$ to $G$ change at position 458 of the human promyostatin cDNA causing the K153R mutation in the protein. E. coli BL21(DE3) cells were transformed with the pPR-IBA2_Promyo_K153R plasmid and protein expression, refolding and purification was performed with the same method that was used for the production of the recombinant wild type human promyostatin [20].

\subsection{Proteolytic digestion of wild type and K153R mutant promyostatin with human furin}

To monitor furin-mediated cleavage of wild type and K153R mutant promyostatins by SDS-PAGE, the recombinant proteins $(1-1 \mu \mathrm{M})$ were digested with human furin $(1 \mu \mathrm{g} / \mathrm{ml})$ in $100 \mathrm{mM}$ Tris- $\mathrm{HCl}, 150 \mathrm{mM} \mathrm{NaCl}, 1 \mathrm{mM} \mathrm{CaCl} 2,100 \mu \mathrm{M}$ PMSF buffer, $\mathrm{pH} 8.0$ at $28^{\circ} \mathrm{C}$. Aliquots were removed at various time points $(0 \mathrm{~h}, 1 \mathrm{~h}$, $4 \mathrm{~h}, 24 \mathrm{~h}$ ) and the composition of the samples was analyzed by SDS-PAGE.

Wild type and K153R mutant latent myostatins were produced by digesting the protein samples $(1 \mu \mathrm{M})$ with $3.5 \mu \mathrm{g} / \mathrm{ml}$ furin in $100 \mathrm{mM}$ Tris- $\mathrm{HCl}, 150 \mathrm{mM} \mathrm{NaCl}, 1 \mathrm{mM} \mathrm{CaCl}_{2}, 100 \mu \mathrm{M}$ PMSF buffer, $\mathrm{pH} 8.0$ at $28^{\circ} \mathrm{C}$ for $24 \mathrm{~h}$. The digestion was arrested with $1 \mu \mathrm{M}$ Furin Inhibitor I; samples were stored at $-20^{\circ} \mathrm{C}$.

\subsection{Proteolytic digestion of wild type and K153R mutant latent myostatin with human BMP-1}

To monitor BMP-1 cleavage of wild type and K153R mutant latent myostatin by SDS-PAGE, latent myostatin preparations $(1 \mu \mathrm{M})$ were digested with $2.5 \mu \mathrm{g} / \mathrm{ml} \mathrm{BMP}-1$ in $25 \mathrm{mM}$ HEPES buffer, pH 7.5 containing $5 \mathrm{mM} \mathrm{CaCl}, 1 \mu \mathrm{M} \mathrm{ZnCl}$ and $1 \mu \mathrm{M}$ Furin Inhibitor I at $37^{\circ} \mathrm{C}$. Aliquots were removed at various time points $(0 \mathrm{~h}, 1 \mathrm{~h}, 4 \mathrm{~h}, 24 \mathrm{~h})$ and the composition of the samples was analyzed by SDS-PAGE.

\subsection{Expression of wild type and K153R mutant promyostatin in HEK 293 human embryonic kidney cells}

The cDNA of promyostatin was first cloned into the pENTRYIBA51 entry vector, then transferred to the pCSG-IBA142 mammalian expression vector according to the instructions of 
the manufacturer. The resulting pCSG-IBA142-Promyo plasmid was used as the template of the Quickchange mutagenesis reaction; mutagenesis was performed with the primers given in Section 2.2 .

HEK 293 cells were transfected with $3 \mu \mathrm{g}$ of either pCSGIBA142-Promyo, pCSG-IBA142-PromyoK153R or pCSG-IBA142control plasmids with Fugene HD transfection reagent according to the recommendations of the manufacturer. $24 \mathrm{~h}$ after transfection the culture media were changed to serum-free DMEM and transient protein expression was allowed for $48 \mathrm{~h}$. The media were concentrated 25-fold and the expressed recombinant proteins were visualized on Western blots using antibody AF1539, specific for the prodomain region of myostatin.

\subsection{Protein analyses}

The concentrations of the recombinant promyostatin proteins were calculated with the extinction coefficient $55640 \mathrm{M}^{-1} \mathrm{~cm}^{-1}$. The extinction coefficient was calculated with the online protein analysis tool PROTPARAM. (http://web.expasy.org/protparam/).

The composition of protein samples was analyzed by SDS-PAGE under both reducing and non-reducing conditions; unless otherwise indicated, the gels were stained with Coomassie Brilliant Blue $\mathrm{G}-250$. For quantitative evaluation of protein compositions the gels were stained with SYPRO Ruby (Bio-Rad Laboratories Hercules CA USA), the stained gels were scanned with the Chemilmager 5500 gel documentation instrument (Alpha Innotech, San Leandro, CA, USA) and the intensities of the protein bands were quantified with the instrument's software.

To compare the rates of furin-mediated cleavage of wild type and K153R mutant promyostatin we monitored the proportion of uncleaved promyostatin chains. The rates of BMP- 1 cleavage of wild type and K153R mutant latent myostatins were compared in a similar way except that in this case we monitored the proportion of uncleaved prodomain chains. Statistical analyses of the data were performed with Student's $t$-test.

\subsection{Reporter assay}

Rhabdomyosarcoma A204 cells were cultured in McCoy's 5A medium supplemented with $10 \%$ fetal bovine serum, penicillin $(100 \mathrm{U} / \mathrm{mL})$ and streptomycin $(100 \mu \mathrm{g} / \mathrm{mL})$ at $37^{\circ} \mathrm{C}, 5 \% \mathrm{CO}_{2}$. $3 \times 10^{4}$ cells were plated in wells of a 96-well plate and were allowed to attach for $24 \mathrm{~h}$. The cells were transiently transfected with 200 ng Luciferase Reporter vector mixture and $0.6 \mu$ l Fugene HD reagent per well. Luciferase Reporter vector mixture contained the SBE4 SMAD responsive firefly luciferase plasmid (Addgene plasmid 16495) and a constitutive Renilla luciferase plasmid in 40:1 ratio [23].

Transfections were performed in McCoy's 5A medium containing $10 \%$ FBS without antibiotics. $24 \mathrm{~h}$ later, the transfection medium was changed to McCoy's $5 \mathrm{~A}$ containing $1 \mathrm{mg} / \mathrm{ml}$ BSA and conditioned for $18 \mathrm{~h}$. McCoy's $5 \mathrm{~A}$ medium containing $1 \mathrm{mg} / \mathrm{ml}$ BSA and different concentrations of recombinant proteins were added to the cells and after $6 \mathrm{~h}$, the cells were washed twice with PBS and lysed using $50 \mu$ passive lysis buffer (Promega Corporation). Firefly and Renilla luciferase activities were measured on an Enspire Multimode Reader (PerkinElmer, Waltham, MA USA). The firefly luciferase units obtained were normalized to the Renilla luciferase units to generate relative luciferase units. Statistical analyses of the data were performed with Student's t-test.

\subsection{Protein modeling}

The homology model of promyostatin was generated with the automated protein structure homology-modeling server
SWISS-MODEL Version 8.05 (http://swissmodel.expasy.org/; [2425]. The template was identified from the SWISS-MODEL template library (SMTL version 2014-07-09, PDB release 2014-07-03) with the automated template search of the pipeline. The model was based on the selected 3rjr.1.A file that contains the structure of porcine proTGFbeta1 [26]. The sequence identity between human myostatin and porcine proTGFbeta 1 was $31.68 \%$ over the aligned region (residues 49-375 of promyostatin). The model generated by the pipeline was analyzed with Swiss-PdbViewer DeepView version 4.01 (http://spdbv.vital-it.ch/; [27]). For further details of homology modeling of human promyostatin see Supplementary file 1.

\section{Results}

\subsection{The K153R mutation does not impair the folding of promyostatin}

K153R promyostatin was expressed in $E$. coli cells and refolded from inclusion bodies according to the protocol described previously [20]. The yields and efficiency of refolding of the mutant protein were indistinguishable from that of the wild type promyostatin, indicating that the replacement of a lysine residue by an arginine at position 153 had no deleterious effect on the structure of promyostatin. This conclusion is also supported by the observation that mutant promyostatin may be activated by furin and BMP-1 to yield mature myostatin (see below). Similarly, our observation that human HEK 293 cells transfected with K153R mutant promyostatin secreted the precursor as efficiently as the wild type protein (see Supplementary file 2) indicates that the mutation has no deleterious effect on the folding and secretion of the protein.

\subsection{The K153R mutation in promyostatin significantly enhances the rate of cleavage by furin}

Wild type and K153R mutant promyostatin were digested with furin and samples were withdrawn after $0 \mathrm{~h}, 1 \mathrm{~h}, 4 \mathrm{~h}$ and $24 \mathrm{~h}$ of digestion and their composition was analyzed by SDS PAGE under both reducing and non-reducing conditions as described in Section 2. As shown in Fig. 1A, furin-digestion of both mutant and wildtype promyostatin proceeds through an intermediate (semipromyostatin) in which only one of the chains of promyostatin dimer is cleaved by furin (non-reduced samples).

The mutant protein, however, is cleaved by furin at a significantly higher rate than the wild type promyostatin. Quantitative evaluation of the gels has shown that the proportion of uncleaved promyostatin decreased at a significantly higher rate in the case of the K153R mutant protein than in the case of the wild type protein (Fig. 1B). Note that at $4 \mathrm{~h}$ and $24 \mathrm{~h}$ the proportion of the uncleaved K153R mutant promyostatin is significantly $\left({ }^{*} P<0.05\right)$ lower than in the case of wild type promyostatin.

\subsection{The K153R mutation does not influence the rate of cleavage of latent myostatin by BMP-1}

Wild type and K153R mutant latent myostatins were digested with BMP- 1 for $0 \mathrm{~h}, 1 \mathrm{~h}, 4 \mathrm{~h}$ and $24 \mathrm{~h}$ and the samples were analyzed by SDS-PAGE as described in Section 2 (Fig. 2A). Quantitative evaluation of the gels has shown that the proportion of uncleaved myostatin prodomain decreased at a similar rate in the case of both the wild type and K153R mutant protein (Fig. 2B). Statistical analysis of the data revealed that the proportion of the uncleaved myostatin prodomain is not significantly different in the case of wild type and mutant myostatin at any of the time points. This 


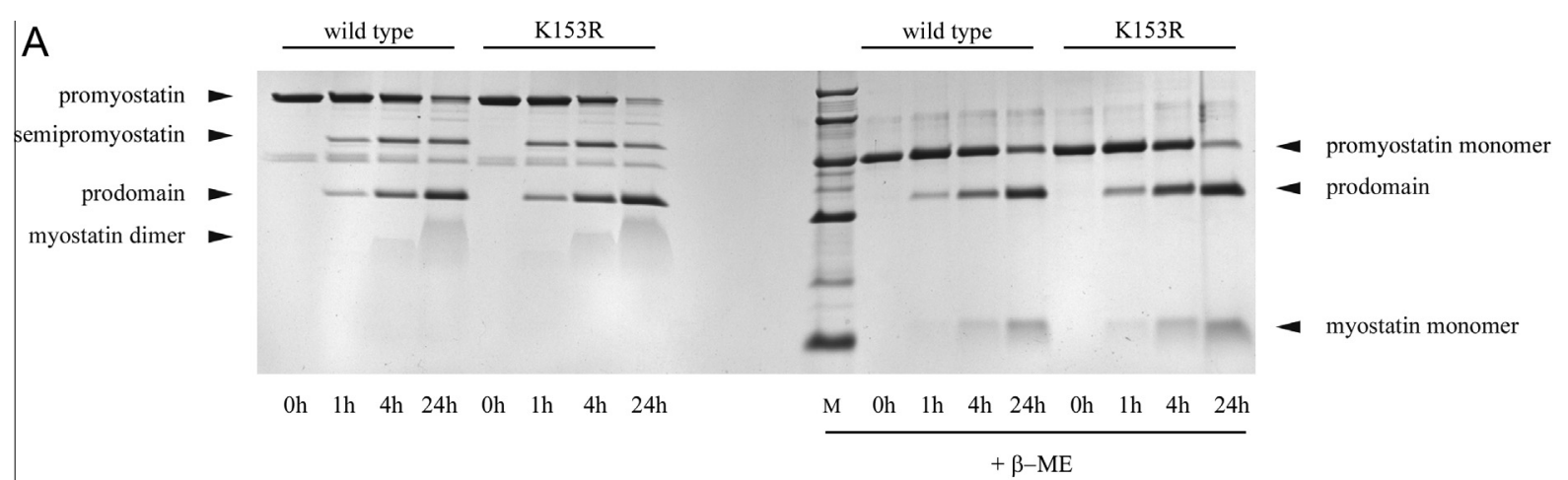

B

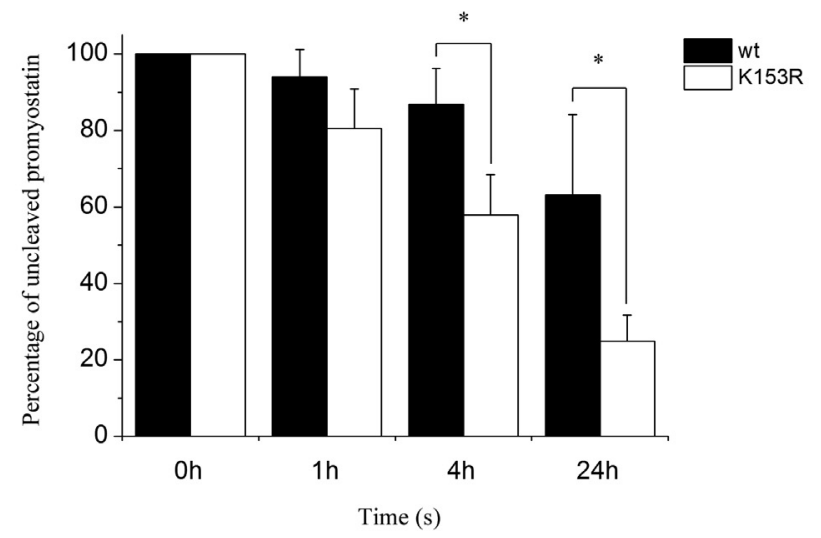

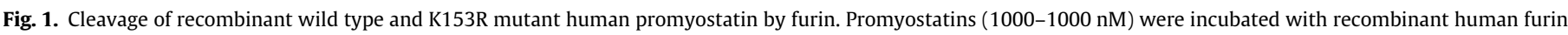

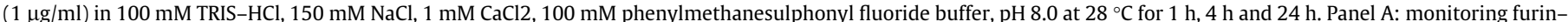

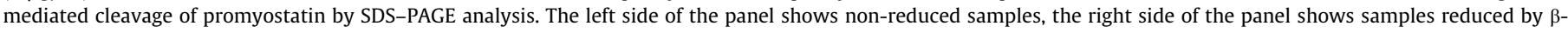

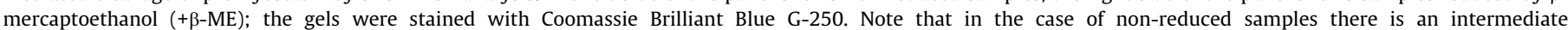

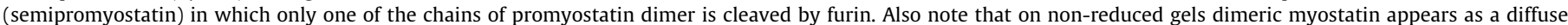

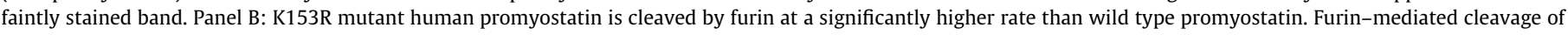

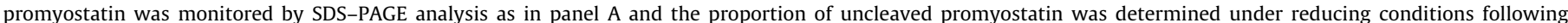

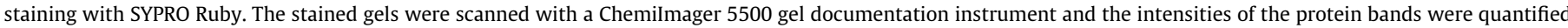

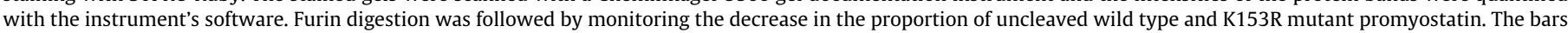

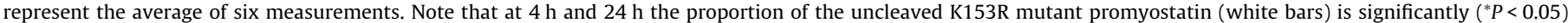
lower than in the case of wild type promyostatin (black bars).

observation suggests that the K153R substitution has no significant effect on BMP-1 cleavage of myostatin prodomain.

\subsection{The K153R mutation does not influence the activity of latent myostatin}

To test whether the K153R mutation has an influence on the activity of the various forms of promyostatin we have monitored myostatin activity in reporter assays as described in Section 2. As shown in Fig. 3, both wild type and K153R mutant promyostatin were devoid of activity: the Relative Luminescence Units were not significantly higher than in the case of control medium.

In harmony with our previous observations [20] wild type latent myostatin had low, but significantly higher $\left({ }^{*} P<0.05\right)$ activity than wild type promyostatin. In this respect, the K153R mutant was similar to wild type: it also had low, but significantly higher $\left({ }^{* *} P<0.01\right)$ activity than mutant promyostatin. The fact that BMP-1 cleavage of both wild type and K153R mutant latent myostatin elicited a similar and significant $\left({ }^{* * *} P<0.001\right)$ increase in myostatin activity is consistent with the observation that the mutant and wild type proteins are equally sensitive to BMP-1 cleavage (see Section 3.3.).

\section{Discussion}

\subsection{Effect of the K153R mutation on the structure of human promyostatin}

In the present work we have shown that the K153R substitution (present in the prodomain region of promyostatin) significantly increases the rate of cleavage by furin, but has no effect on the activity of latent myostatin or on the rate of its cleavage by BMP1. The most plausible explanation for such a specific effect on furin-cleavage is that the substitution of the Lys-153 residue by an arginine residue does not cause a gross alteration in the overall structure of promyostatin, but might cause more subtle changes that affect furin-cleavage.

Although, the three-dimensional structure of promyostatin is unknown, its homology with pro-TGF- $\beta 1$ permitted the construction of a homology model based on the known crystal structure of the latter protein [26]. Based on sequence alignments Shi et al. [26] have suggested that prodomains of TGF- $\beta$ family members have similar folds. Of particular interest is the fact that myostatin is a relatively close homolog of TGF- $\beta 1$, permitting the assumption that the secondary structural elements and their spatial arrangement are similar in these proteins [26]. 


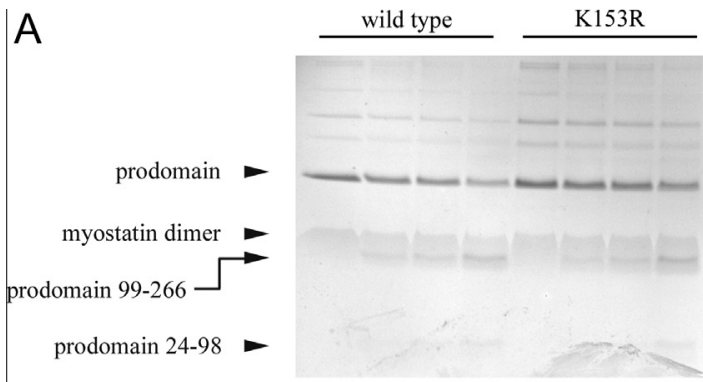

$0 \mathrm{~h} \quad 1 \mathrm{~h} \quad 4 \mathrm{~h} \quad 24 \mathrm{~h} \quad 0 \mathrm{~h} \quad 1 \mathrm{~h} \quad 4 \mathrm{~h} \quad 24 \mathrm{~h}$

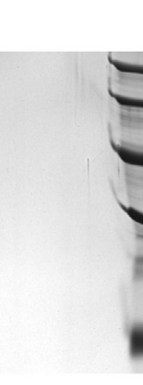

M $\quad 0 \mathrm{~h} \quad 1 \mathrm{~h} \quad 4 \mathrm{~h} \quad 24 \mathrm{~h} \quad 0 \mathrm{~h} \quad 1 \mathrm{~h} \quad 4 \mathrm{~h} \quad 24 \mathrm{~h}$

$+\beta-\mathrm{ME}$

B

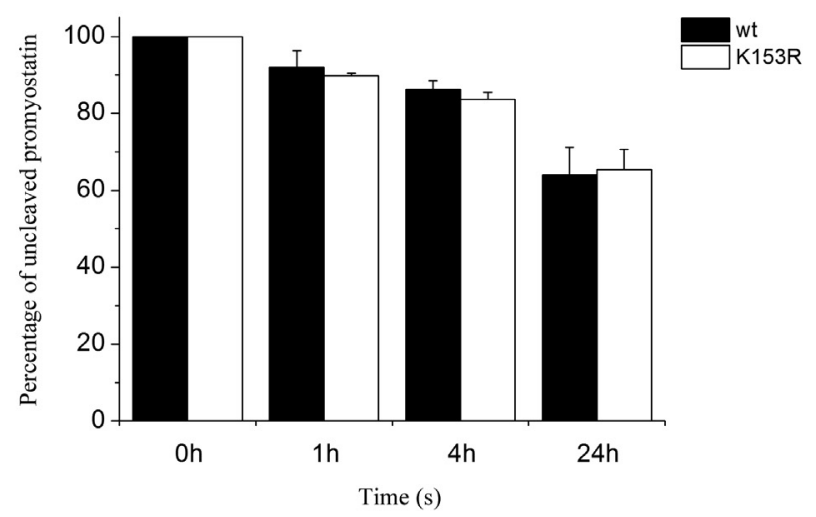

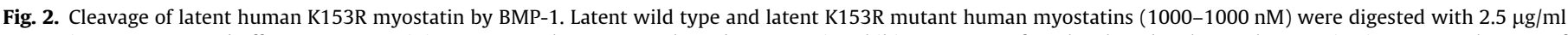

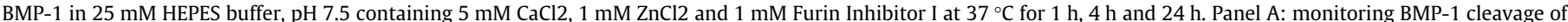

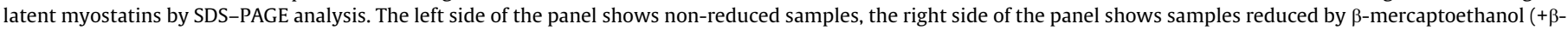

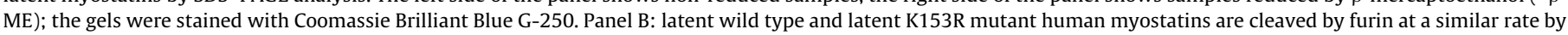

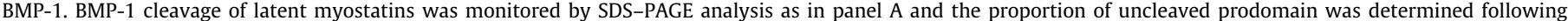

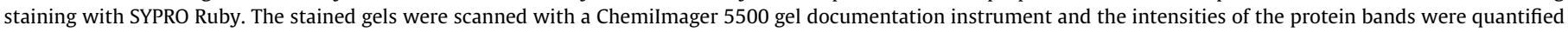

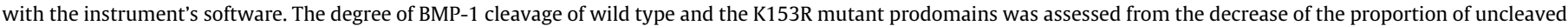

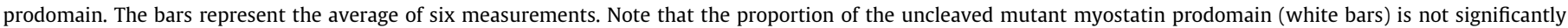
different in the case of wild type and mutant myostatin (black bars) at any of the time points.

In the crystal structure of pro-TGF- $\beta 1$ (and the homology model of myostatin, Fig. 4), the N-terminal part of the prodomain provides the 'straitjacket' that interacts with the growth-factor monomer. The 'straitjacket' consists of the $\alpha 1$ helix and the 'latency lasso', the coiled linker region that connects the $\alpha 1$ and $\alpha 2$ helices of the prodomain. Our observation that substitution of Lys-153 by an arginine does not affect the activity of latent myostatin is consistent with the fact that Lys-153 is relatively distant from the $\alpha 1$ helix-growth factor interaction (Fig. 4). Similarly, the observation that substitution of Lys-153 by an arginine does not affect BMP-1 cleavage of latent myostatin is in harmony with the fact that Lys-153 is remote from the BMP-1 cleavage site (Fig. 4).

In the crystal structure of pro-TGF- $\beta 1$ no coordinates were recorded for the long, flexible prodomain-growth-factor connection (http://www.rcsb.org/pdb/explore.do?structureId=3RJR), therefore the structure of this linker in the homology model is uncertain. Nevertheless, we note with interest that this flexible linker region, that contains the RXRR furin recognition motif (Arg263-Ser264Arg265-Arg266), is relatively close to Lys-153 (Fig. 4), suggesting that replacement of Lys-153 by an arginine residue may increase the accessibility of the cleavage site for furin. However, it is also possible that the effect of the K153 $\rightarrow$ R153 substitution on furincleavage reflects the known affinity of furin for polyarginines [27]. Studies on the crystal structures of furin [28-30] revealed that the targeting of substrates is dominated by extended electro- static interactions with 18 clustered negatively charged residues in the substrate binding cleft of the enzyme and that this extended site may bind arginines not only at the at the S1, S2 and S3 sites, but also at the $S 5$ and $S 6$ subsites. It seems possible that in the case of the K153R mutant of promyostatin the side chain of Arg-153 may also contribute to the recognition of the cleavage-site by furin, and this could explain why this mutant is more susceptible to furin cleavage.

\subsection{Effect of the K153R mutation on human phenotype}

Since the K153R mutation makes promyostatin more susceptible to furin-cleavage, the mutation may facilitate the formation of latent myostatin in individuals carrying this mutation. We have shown that both wild type [19] and K153R mutant (present study) latent myostatin have significant activity, therefore elevation in the levels of latent myostatin results in myostatin activity even in the absence of BMP-1 cleavage. On the basis of these considerations it is plausible to assume that individuals carrying the K153R mutation may have higher myostatin activity than individuals with the wild type genotype. Since myostatin is a negative regulator of muscle mass [4] and a positive regulator of adipose tissues $[10,11]$ increased myostatin activity may provide an explanation as to why the K153R polymorphism is associated with lower muscle strength and obesity. 


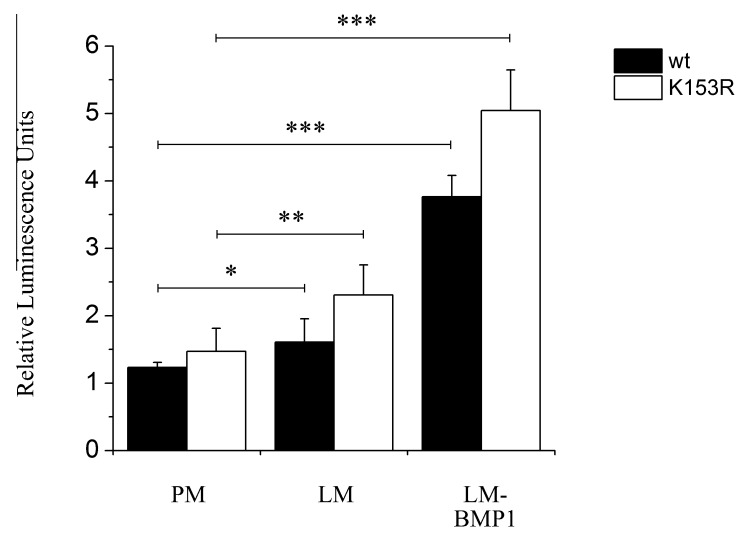

Fig. 3. Luciferase reporter assay of myostatin activity of wild type and K153R mutant human promyostatin and their derivatives. Rhabdomyosarcoma A204 cells were transiently transfected with the SMAD Luciferase reporter vector and a Renilla luciferase vector, and incubated for $16 \mathrm{~h}$ with different forms of myostatin ( $5 \mathrm{nM}$ ). Firefly luciferase units were normalized to Renilla luciferase units. The activities of promyostatins and their derivatives are expressed in Relative luminescence units, relative to the luminescence observed with the control medium. PM: promyostatin; LM: latent myostatin; LM-BMP1: latent myostatin digested with BMP-1. The bars represent the average of six parallel experiments with the wild type (black bars) and K153R mutant proteins (white bars). Note that both wild type and mutant latent myostatin have significantly higher activity than the corresponding promyostatins even in the absence of BMP- 1 cleavage. Values are means \pm standard errors. ${ }^{*} P<0.05$ versus control samples; ${ }^{* *} P<0.01$ versus control samples ${ }^{* * *} P<0.001$ versus control samples.

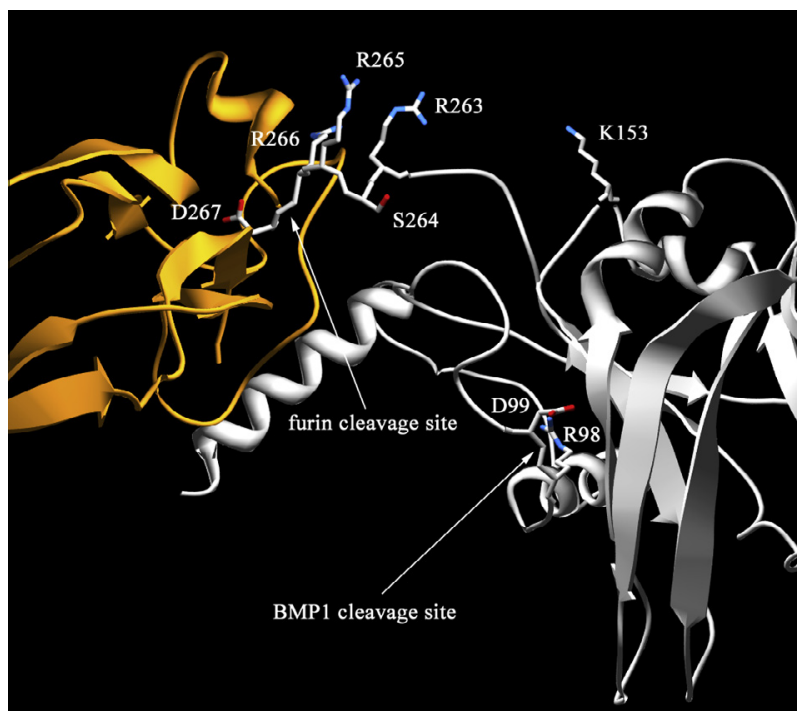

Fig. 4. Protein modeling of the 3D structure of human promyostatin. The figure shows the model of human promyostatin highlighting the position of the residues adjacent to the BMP-1 cleavage site (residues Arg-98/Asp-99), the furin-cleavage site (Arg263-Ser264-Arg265-Arg266/Asp267) and the position of Lys-153 that is replaced by an Arg in the K153R mutant myostatin. The growth factor domain of promyostatin is shown in gold. The homology model was computed using the structure of pro-TGF- $\beta 1$ as a template.

The molecular basis of the association of the K153R mutation with extreme longevity is less clear but the most parsimonious explanation is that it is also due to increased myostatin activity. It is noteworthy in this respect that recent studies have directly implicated the fly ortholog of myostatin, myoglianin, in controlling lifespan $[21,22]$. These authors have shown that myoglianin overexpression in flies extends lifespan, whereas myoglianin RNAi has converse effects, leading them to suggest that myostatin may have a similar role in regulating lifespan in mammals. The association of the K153R mutation (and its increased myostatin activity) with extreme longevity in humans is in harmony with this hypothesis.

Garatachea et al. [19] suggested that the beneficial effect of the $\mathrm{R}$ allele on exceptional longevity is based on the inhibitory role that myostatin plays in the mTOR signaling pathway. The strongest argument in favor of this interpretation is that the most robust improvements in ageing are caused by dietary restriction and genetic down-regulation of nutrient-sensing pathways, such as the mTOR pathway [31,32]. However, it is also possible that association of the K153R mutation of promyostatin with longevity is less direct: myostatin might exert its effect through its influence on skeletal muscle that in turn controls systemic aging and lifespan [33].

\section{Acknowledgements}

This work was supported by Grants 72125 and 108630 of the National Scientific Research Fund of Hungary (OTKA).

\section{Appendix A. Supplementary data}

Supplementary data associated with this article can be found, in the online version, at http://dx.doi.org/10.1016/j.febslet.2014. 12.011 .

\section{References}

[1] Anderson, S.B., Goldberg, A.L. and Whitman, M. (2008) Identification of a novel pool of extracellular pro-myostatin in skeletal muscle. J. Biol. Chem. 283, 7027-7035.

[2] Lee, S.J. and McPherron, A.C. (2001) Regulation of myostatin activity and muscle growth. Proc. Natl. Acad. Sci. U.S.A. 98, 9306-9311.

[3] Wolfman, N.M., McPherron, A.C., Pappano, W.N., Davies, M.V., Song, K., Tomkinson, K.N., Wright, J.F., Zhao, L., Sebald, S.M., Greenspan, D.S. and Lee S.J. (2003) Activation of latent myostatin by the BMP-1/tolloid family of metalloproteinases. Proc. Natl. Acad. Sci. U.S.A. 100, 15842-15846.

[4] McPherron, A.C., Lawler, A.M. and Lee, S.J. (1997) Regulation of skeletal muscle mass in mice by a new TGF-beta superfamily member. Nature 387 83-90.

[5] Szabó, G., Dallmann, G., Müller, G., Patthy, L., Soller, M. and Varga, L. (1998) A deletion in the myostatin gene causes the compact (Cmpt) hypermuscular mutation in mice. Mamm. Genome 9, 671-672.

[6] Grobet, L., Martin, L.J., Poncelet, D., Pirottin, D., Brouwers, B., Riquet, J., Schoeberlein, A., Dunner, S., Menissier, F., Massabanda, J., Fries, R., Hanset, R. and Georges, M. (1997) A deletion in the bovine myostatin gene causes the double-muscled phenotype in cattle. Nat. Genet. 17, 71-74.

[7] Kambadur, R., Sharma, M., Smith, T.P. and Bass, J.J. (1997) Mutations in myostatin (GDF8) in double-muscled Belgian Blue and Piedmontese cattle. Genome Res. 7, 910-916.

[8] McPherron, A.C. and Lee, S.J. (1997) Double muscling in cattle due to mutations in the myostatin gene. Proc. Natl. Acad. Sci. U.S.A. 94, 12457-12461.

[9] Schuelke, M., Wagner, K.R., Stolz, L.E., Hubner, C., Riebel, T., Kömen, W., Braun, T., Tobin, J.F. and Lee, S.J. (2004) Myostatin mutation associated with gross muscle hypertrophy in a child. N. Engl. J. Med. 350, 2682-2688.

[10] McPherron, A.C. and Lee, S.J. (2002) Suppression of body fat accumulation in myostatin-deficient mice. J. Clin. Invest. 109, 595-601.

[11] Lin, J., Arnold, H.B., Della-Fera, M.A., Azain, M.J., Hartzell, D.L. and Baile, C.A. (2002) Myostatin knockout in mice increases myogenesis and decreases adipogenesis. Biochem. Biophys. Res. Commun. 291, 701-706.

[12] Guo, T., Jou, W., Chanturiya, T., Portas, J., Gavrilova, O. and McPherron, A.C. (2009) Myostatin inhibition in muscle, but not adipose tissue, decreases fat mass and improves insulin sensitivity. PLoS One 4 (3), e4937.

[13] Ferrell, R.E., Conte, V., Lawrence, E.C., Roth, S.M., Hagberg, J.M. and Hurley, B.F. (1999) Frequent sequence variation in the human myostatin (GDF8) gene as a marker for analysis of muscle-related phenotypes. Genomics 62, 203-207.

[14] Saunders, M.A., Good, J.M., Lawrence, E.C., Ferrell, R.E., Li, W.H. and Nachman, M.W. (2006) Human adaptive evolution at myostatin (GDF8), a regulator of muscle growth. Am. J. Hum. Genet. 79, 1089-1097.

[15] Seibert, M.J., Xue, Q.L., Fried, L.P. and Walston, J.D. (2001) Polymorphic variation in the human myostatin (GDF-8) gene and association with strength measures in the Women's Health and Aging Study II cohort. J. Am. Geriatr. Soc. 49, 1093-1096.

[16] González-Freire, M., Rodríguez-Romo, G., Santiago, C., Bustamante-Ara, N. Yvert, T., Gómez-Gallego, F., Serra Rexach, J.A., Ruiz, J.R. and Lucia, A. (2010) The K153R variant in the myostatin gene and sarcopenia at the end of the human lifespan. Age (Dordr) 32, 405-409. 
[17] Santiago, C., Ruiz, J.R., Rodríguez-Romo, G., Fiuza-Luces, C., Yvert, T., GonzalezFreire, M., Gómez-Gallego, F., Morán, M. and Lucia, A. (2011) The K153R polymorphism in the myostatin gene and muscle power phenotypes in young, non-athletic men. PLoS One 6 (1), e16323.

[18] Bhatt, S.P. Nigam, P. Misra, A., Guleria, R., Luthra, K. Jain, S.K. and Qadar Pasha, M.A. (2012) Association of the myostatin gene with obesity, abdomina obesity and low lean body mass and in non-diabetic Asian Indians in north India. PLoS One 7 (8), e40977.

[19] Garatachea, N., Pinós, T., Cámara, Y., Rodríguez-Romo, G., Emanuele, E., Ricevuti, G., Venturini, L., Santos-Lozano, A., Santiago-Dorrego, C., Fiuza-Luces, C., Yvert, T., Andreu, A.L. and Lucia, A. (2013) Association of the K153R polymorphism in the myostatin gene and extreme longevity. Age (Dordr) 35, 2445-2454.

[20] Szláma, G., Trexler, M. and Patthy, L. (2013) Latent myostatin has significan activity and this activity is controlled more efficiently by WFIKKN1 than by WFIKKN2. FEBS J. 280, 3822-3839.

[21] Demontis, F., Patel, V.K., Swindell, W.R. and Perrimon, N. (2014) Intertissue control of the nucleolus via a myokine-dependent longevity pathway. Cell Rep. 7, 1481-1494.

[22] Patel, V.K. and Demontis, F. (2014) GDF11/myostatin and aging. Aging (Albany NY) 6, 351-352

[23] Zawel, L., Dai, J.L., Buckhaults, P., Zhou, S., Kinzler, K.W., Vogelstein, B. and Kern, S.E. (1998) Human Smad3 and Smad4 are sequence-specific transcription activators. Mol. Cell 1, 611-617.

[24] Arnold, K., Bordoli, L., Kopp, J. and Schwede, T. (2006) The SWISS-MODEL workspace: a web-based environment for protein structure homology modelling. Bioinformatics 22, 195-201.
[25] Biasini, M., Bienert, S., Waterhouse, A., Arnold, K., Studer, G., Schmidt, T., Kiefer, F., Cassarino, T.G., Bertoni, M., Bordoli, L. and Schwede, T. (2014) SWISSMODEL: modelling protein tertiary and quaternary structure using evolutionary information. Nucleic Acids Res. 42 (Web Server issue), W252258.

[26] Shi, M., Zhu, J., Wang, R., Chen, X., Mi, L., Walz, T. and Springer, T.A. (2011) Latent TGF- $\beta$ structure and activation. Nature 474, 343-349.

[27] Guex, N. and Peitsch, M.C. (1997) SWISS-MODEL and the Swiss-PdbViewer: an environment for comparative protein modeling. Electrophoresis 18, 27142723.

[28] Cameron, A., Appel, J., Houghten, R.A. and Lindberg, I. (2000) Polyarginines are potent furin inhibitors. J. Biol. Chem. 275. 36741-36419.

[29] Henrich, S., Cameron, A., Bourenkov, G.P., Kiefersauer, R., Huber, R., Lindberg, I., Bode, W. and Than, M.E. (2003) The crystal structure of the proprotein processing proteinase furin explains its stringent specificity. Nat. Struct. Biol. $10,520-526$.

[30] Dahms, S.O., Hardes, K., Becker, G.L., Steinmetzer, T., Brandstetter, H. and Than, M.E. (2014) X-ray structures of human furin in complex with competitive inhibitors. ACS Chem. Biol. 9, 1113-1118.

[31] Fontana, L., Partridge, L. and Longo, V.D. (2010) Extending healthy life spanfrom yeast to humans. Science 328, 321-326.

[32] Kenyon, C.J. (2010) The genetics of ageing. Nature 464, 504-512.

[33] Demontis, F., Piccirillo, R., Goldberg, A.L. and Perrimon, N. (2013) The influence of skeletal muscle on systemic aging and lifespan. Aging Cell 12, 943-949. 